\title{
Scalable quantum simulation of pulsed entanglement and Einstein-Podolsky-Rosen steering in optomechanics
}

\author{
S. Kiesewetter ${ }^{1}$, Q. Y. He ${ }^{1}$, P. D. Drummond ${ }^{1}$, and M. D. Reid ${ }^{1}$ \\ ${ }^{1}$ Centre for Quantum Atom Optics, \\ Swinburne University of Technology, Melbourne, Australia
}

\begin{abstract}
We demonstrate a complete, probabilistic quantum dynamical simulation of the standard nonlinear Hamiltonian of optomechanics, including decoherence at finite temperatures. Robust entanglement of an optical pulse with the oscillator is predicted, as well as strong quantum steering between the optical and mechanical systems. Importantly, our probabilistic quantum simulation method uses the positive-P technique, which is scalable to large Hilbert spaces.
\end{abstract}

Introduction. Optomechanical oscillators provide a fundamental test of mesoscopic quantum mechanics, as well as having potential technological applications in a wide variety of sensitive measurements. Impressive success in cooling optomechanical systems near their ground state has been reported experimentally [1], resulting in the demonstration of a number of quantum mechanical effects for mesoscopic systems [5,8]. An outstanding goal is to observe quantum correlations, such as entanglement and the nonlocality predicted by Einstein, Podolsky and Rosen (EPR) [9, 10] for macroscopic, massive objects [11 14. In the first instance, it is of interest whether an optical field can entangle with a massive oscillator, and whether the two systems can show the strange directional "spooky action-at-a-distance" [15] effects that Schrodinger called "steering" [16-18.

In this paper, we carry out the first scalable, probabilistic quantum mechanical simulations of the standard nonlinear optomechanical Hamiltonian. This helps to unravel the quantum mechanical interplay between entanglement generation, created by the nonlinear radiation coupling, and the thermal decoherence due to reservoirs. We use this to study the dynamical generation of entanglement and EPR-steering correlations, for pulsed inputs and realistic experimental parameters. Models used previously often make numerous assumptions, ranging from linearization [12] to adiabatic approximations [19], or both. While very useful in giving analytic results, it unclear how justified these assumptions are. In our simulations we utilize the exact positive-P phase-space method [20], which exists as a positive probability distribution for all quantum states.

The main limitation in current optomechanics experiments is that long interaction times lead to increased decoherence, owing to a coupling to the environmental heat bath which is often at relatively high temperature. The use of pulsed probes can overcome this, and it was recently proposed[19 to create and verify entanglement with two successive pulses of light so that interactions can take place on fast time scales [21, 22]. This theoretical treatment was restricted to an adiabatic, linearized study using symmetric entanglement measures
[23]. They showed that entanglement is feasible, provided $Q f \gg k_{B} T_{\text {bath }} / h$, where $T_{\text {bath }}$ is the temperature of the environment, $f$ is the frequency of mechanical oscillator and $Q$ is the cavity quality. By comparison, our method can treat arbitrary pulse shapes, is not linearized, and uses optimized measures for entanglement and EPR. We compare our results with the truncated Wigner method [24 26], valid for large photon-numbers [27-29].

This simulation technique, which has been used on other parametric problems [30, has no approximations apart from those inherent in the standard model [41. It successfully produces results for realistic experimental parameters at large photon number. Compared to direct diagonalization [31] or quantum trajectory approaches [32], the method readily scales to large Hilbert spaces [34. Neither approximations [33 nor new hardware 35] are required, apart from sampling error issues with highorder correlations [36. Therefore, it has excellent potential for treat the challenging new generation of multimode optomechanical crystal devices 37 .

The results of this full analysis validate some of the earlier predictions for the simplified model in Refs. [19, 38]. It is directly useful for addressing experiments with optomechanical systems. Our main finding is that entanglement of pulse and oscillator mode proves to be robust, without requiring a low temperature reservoir, but that the thermal noise provides a stronger barrier to the EPR steering paradox than to entanglement. The thermal barrier acts directionally, to prevent steering of the mechanical system when thermally excited. This is a fundamentally asymmetric manifestation of nonlocality, beautifully illustrated by the oscillator-pulse system.

Hamiltonian and stochastic equations. We consider the standard, single-mode model [39 41] for an optomechanical Fabry-Perot cavity with coherent pumping and damping. The Hamiltonian includes the energy of the cavity mode at angular frequency $\omega_{c}$, the mechanical oscillator mode at $\omega_{m}$, and an input driving the cavity mode with amplitude $E(t)$ :

$$
\begin{aligned}
\hat{H} / \hbar= & \omega_{c} \hat{a}^{\dagger} \hat{a}+\omega_{m} \hat{b}^{\dagger} \hat{b}+\chi_{0} \hat{a}^{\dagger} \hat{a}\left(\hat{b}+\hat{b}^{\dagger}\right) \\
& +i E(t)\left(\hat{a}^{\dagger} e^{-i \omega_{l} t}-\hat{a} e^{i \omega_{l} t}\right)+\hat{H}_{r},
\end{aligned}
$$




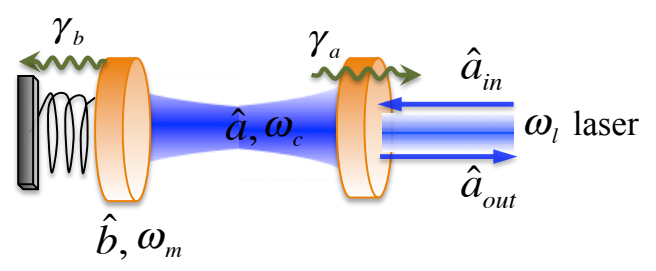

Figure 1. Schematic diagram of a driven optomechanical system. Here, $\hat{a}_{\text {in }}$ and $\hat{a}_{\text {out }}$ denote ingoing and outgoing fields, while $\hat{a}$ is an annihilation operator of the optical mode with resonance frequency $\omega_{c}$, and $\hat{b}$ corresponds to a mechanical mode with oscillation frequency $\omega_{m}$. A light pulse of duration $\tau$ and carrier frequency $\omega_{l}$ impinges on the cavity and interacts with the mirror via radiation pressure.

The first two terms give the energy of the cavity field and the mechanical oscillator, the third term denotes the optomechanical interaction, where $\chi_{0}$ is the singlephoton coupling, and the fourth is the coupling to the coherent input field. $\hat{H}_{r}$ describes dissipation of the two subsystems via coupling to reservoirs. The system is driven by a light pulse of duration $\tau$ and carrier frequency $\omega_{l}=\omega_{c}-\Delta$, with $N_{p h}$ photons. The laser driving strength is $E(t)=E_{0} \varepsilon(t)=\sqrt{2 \gamma_{a} N_{p h}} \varepsilon(t)$, and the envelope function $\varepsilon(t)$ is normalized so that $\int_{0}^{\tau} d t|\varepsilon(t)|^{2}=1$. A diagram is shown in Fig. 1 .

This is a driven open system, hence the density matrix must be calculated as the solution of a master equation. For simplicity, we transform to a rotating frame in which the free-field time evolution of the field operators is removed, where $\Delta=\omega_{c}-\omega_{l}$ is the detuning of the laser driving frequency with respect to the cavity resonance. The master equation for the reduced density operator is then given by

$$
\begin{aligned}
\frac{d \hat{\rho}}{d t}= & -i\left[\Delta \hat{a}^{\dagger} \hat{a}+\omega_{m} \hat{b}^{\dagger} \hat{b}+\chi_{0} \hat{a}^{\dagger} \hat{a}\left(\hat{b}+\hat{b}^{\dagger}\right)+i E(t)\left(\hat{a}^{\dagger}-\hat{a}\right), \hat{\rho}\right] \\
& +\sum_{i=1,2} \gamma_{i}\left(\bar{n}_{i, t h}+1\right)\left(2 \hat{a}_{i} \hat{\rho} \hat{a}_{i}^{\dagger}-\hat{a}_{i}^{\dagger} \hat{a}_{i} \hat{\rho}-\hat{\rho} \hat{a}_{i}^{\dagger} \hat{a}_{i}\right) \\
& +\sum_{i=1,2} \gamma_{i} \bar{n}_{i, t h}\left(2 \hat{a}_{i}^{\dagger} \hat{\rho} \hat{a}_{i}-\hat{a}_{i} \hat{a}_{i}^{\dagger} \hat{\rho}-\hat{\rho} \hat{a}_{i} \hat{a}_{i}^{\dagger}\right) .
\end{aligned}
$$

where $\gamma_{a}$ and $\gamma_{b}$ are the cavity decay rate and mechanical dissipation rate. Here the vector $\hat{\boldsymbol{a}}=\left(\hat{a}_{1}, \hat{a}_{2}\right)=(\hat{a}, \hat{b})$ is introduced for convenience, with $i=1,2 \sim a, b$ indexing the optical and mechanical modes respectively.

We transcribe this master equation into a $c$-number phase space evolution equation, with similar features to related equations found in optical fibre simulations [27. First we use the positive-P representation [20], which gives an exact, positive phase-space representation of any quantum state (2). This method has a normally-ordered quantum-stochastic correspondence, with $\left\langle\alpha^{+} \alpha\right\rangle_{S}=\left\langle\hat{a}^{\dagger} \hat{a}\right\rangle_{Q},\left\langle\beta^{+} \beta\right\rangle_{S}=\left\langle\hat{b}^{\dagger} \hat{b}\right\rangle_{Q}$. After obtaining a Fokker-Planck equation, one obtains a set of four complex Itô stochastic equations:

$$
\begin{aligned}
d \alpha & =\left\{E(t)-\left[i \Delta+i \chi_{0}\left(\beta+\beta^{+}\right)+\gamma_{a}\right] \alpha\right\} d t+d W_{a}, \\
d \beta & =\left[-\left(i \omega_{m}+\gamma_{b}\right) \beta-i \chi_{0} \alpha \alpha^{+}\right] d t+d W_{b}, \\
d \alpha^{+} & =\left\{E^{*}(t)+\left[i \Delta+i \chi_{0}\left(\beta+\beta^{+}\right)-\gamma_{a}\right] \alpha^{+}\right\} d t+d W_{a}^{+}, \\
d \beta^{+} & =\left[\left(i \omega_{m}-\gamma_{b}\right) \beta^{+}+i \chi_{0} \alpha \alpha^{+}\right] d t+d W_{b}^{+},
\end{aligned}
$$

The noises here are due to both internal nonlinearities and thermal noise inputs, so that generically $d W_{i}=$ $d W_{i}^{\chi}+\sqrt{2 \gamma_{i}} d W_{i}^{i n}$, whose non-vanishing correlations are: $\left\langle d W_{i}^{i n} d W_{i}^{i n+}\right\rangle=\bar{n}_{i, t h} d t,\left\langle d W_{i}^{\chi} W_{j}^{\chi}\right\rangle=-i \delta_{i, 3-j} \chi_{0} \alpha d t$, and $\left\langle d W_{i}^{\chi+} W_{j}^{\chi+}\right\rangle=i \delta_{i, 3-j} \chi_{0} \alpha^{+} d t$.

As an alternative approach which is simpler - but approximate - we can use the truncated Wigner distribution 24-26], which is a symmetrically ordered representation. After removing higher-order derivatives in the Fokker-Planck equation (in an approximation valid at large photon number), we obtain the equations:

$$
\begin{aligned}
d \alpha & =\left\{E(t)-\left[i \Delta+i \chi_{0}\left(\beta+\beta^{*}\right)+\gamma_{a}\right] \alpha\right\} d t+\sqrt{2 \gamma_{a}} d W_{a}^{i n}, \\
d \beta & =\left[-\left(i \omega_{m}+\gamma_{b}\right) \beta-i \chi_{0}|\alpha|^{2}\right] d t+\sqrt{2 \gamma_{b}} d W_{b}^{i n} .
\end{aligned}
$$

Here there are two complex variables, with non vanishing noise correlations are given by $\left\langle d W_{i}^{i n}\right\rangle=0$, $\left\langle d W_{i}^{i n} d W_{i}^{i n *}\right\rangle=\left(\bar{n}_{i, t h}+1 / 2\right) d t$, where $i=a, b$, and $\bar{n}_{i, t h}$ are the mean heat bath occupations. These equations imply that $\left\langle\alpha \alpha^{\dagger}\right\rangle_{S}=\langle\hat{n}+1 / 2\rangle_{Q}=1 / 2$ when there is no driving or coupling, as required for a vacuum state.

In the simulations, we drive the cavity with a bluedetuned laser pulse where the resonant scattering to the lower (Stokes) sideband $\left(\omega_{c}=\omega_{l}-\omega_{m}\right.$, i.e. $\left.\Delta=-\omega_{m}\right)$ enhances entanglement. We choose parameters that correspond to recent experiments on $S i$ optomechanical crystal structures [2, with $\omega_{m} / 2 \pi=3.7 G H z, Q_{m}=$ $\omega_{m} / \gamma_{b}=10^{5}, \gamma_{b} / 2 \pi=37 \mathrm{KHz}, \gamma_{a} / 2 \pi=0.26 \mathrm{GHz}$, $\chi_{0} / 2 \pi=910 K \mathrm{~Hz}$. The pulse parameters used a total photon number of $N_{p h}=8.2 \times 10^{6}$ and a pulse duration of $\tau=0.04 \mu \mathrm{s}$. We present results for square pulses here, although a variety of pulse shapes $\varepsilon(t)$, ranging from square waves to Gaussians, all gave strong entanglement and steering. We will describe these more general results elsewhere, for space reasons.

Two different heat bath temperatures were chosen for comparison purposes: either with a cold reservoir at $T_{\text {bath }}=200 \mathrm{mK}$, or a hot reservoir of $T_{b a t h}=20 \mathrm{~K}$ as in recent experiments. In both cases, the initial mechanical occupation number was chosen as $n_{b, 0}=0.7$, corresponding to an initial oscillator temperature of $200 \mathrm{mK}$. This is typically obtained using laser pre-cooling of the mechanical oscillator.

To study the optical properties of the output field, it is convenient to use operators in a frame rotating with $\omega_{m}$, by utilizing $\hat{a}^{r}=\hat{a} e^{-i \omega_{m} t}$ and $\hat{b}^{r}=\hat{b} e^{i \omega_{m} t}$, with similar definitions for the input and output operators. A normalized output mode in the rotating frame is obtained using the standard cavity input-output relations, 
$\hat{a}_{\text {out }}(t)+\hat{a}_{\text {in }}(t)=\sqrt{2 \gamma_{a}} \hat{a}^{r}$ [42, 43], together with a mode function designed to match the gain characteristics of the cavity [19]. We define

$$
\hat{A}_{\text {out }}=\sqrt{\frac{1}{\mathcal{N}(\tau)}} \int_{0}^{\tau} d t e^{r(t)} \hat{a}_{\text {out }}(t),
$$

in terms of the integrated gain, $r(t)=\int_{0}^{t} G\left(t^{\prime}\right) d t^{\prime}$ and normalization, $\mathcal{N}(\tau)=\int_{0}^{\tau} e^{2 r(t)} d t$, where $G(t)=$ $\sqrt{\chi_{0}^{2} E(t) / \gamma_{a}\left(\Delta^{2}+\gamma_{a}^{2}\right)}$ is the effective optomechanical coupling. This causes the mirror motion to become correlated with a light mode of central frequency $\omega_{c}=\omega_{l}-\omega_{m}$.

In either representation, the corresponding stochastic equation for the output field is

$$
d \alpha_{\text {out }}=\sqrt{\frac{1}{\mathcal{N}(\tau)}} e^{r(t)}\left(\sqrt{2 \gamma_{a}} \alpha^{r} d t-d W_{a}^{i n, r}\right) .
$$

Robust asymmetric entanglement - The measured output covariance of a general quadrature $\hat{X}_{c}^{\theta, \text { out }}=$ $\left(e^{-i \theta} \hat{A}_{\text {out }}(\tau)+e^{i \theta} \hat{A}_{\text {out }}^{\dagger}(\tau)\right) / 2, \quad \hat{X}_{m}^{\varphi, \text { out }}=\left(e^{-i \varphi} \hat{b}(\tau)+\right.$ $\left.e^{i \varphi} \hat{b}^{\dagger}(\tau)\right) / 2$ can be written as

$$
V_{c m}^{\theta, \varphi}=\left\langle\Delta \hat{X}_{c}^{\theta} \Delta \hat{X}_{m}^{\varphi}\right\rangle
$$

Here the fluctuations $\Delta \hat{X}_{c, m}^{\theta, \varphi}$ are defined as $\Delta \hat{X}_{c, m}^{\theta, \varphi}=$ $\hat{X}_{c, m}^{\theta, \varphi}-\left\langle\hat{X}_{c, m}^{\theta, \varphi}\right\rangle$, where $\theta, \varphi$ are the phase angles for a phase-sensitive local oscillator measurement. We then calculate continuous variable entanglement and EPR correlations between the output optical field quadrature and the position and momentum of the resonator. The optimal phase to obtain the strongest signature of entanglement is $\theta, \varphi=0, \pi / 2$.

We first consider the entanglement signatures. This is best indicated using asymmetric weightings of the quadratures $(\hbar=1 / 2)[38$

$\Delta_{g, \text { ent }}=\frac{\left[\Delta\left(\hat{X}_{m}^{\text {out }}+g_{x} \hat{X}_{c}^{\text {out }}\right)\right]^{2}+\left[\Delta\left(\hat{P}_{m}^{\text {out }}+g_{p} \hat{P}_{c}^{\text {out }}\right)\right]^{2}}{\left(1+g_{x} g_{p}\right) / 2}<$

where $g_{x}, g_{p}$ are real gains used in post-processing the data. In this system, the $\Delta_{g, e n t}$ can be minimized by choosing the optimal gain factor $g_{x, p}=g$

$$
g=\frac{-b+\sqrt{b^{2}-4 a c}}{2 a},
$$

where $c=\left\langle\hat{X}_{m}^{\text {out }}, \hat{X}_{c}^{\text {out }}\right\rangle+\left\langle\hat{X}_{c}^{\text {out }}, \hat{X}_{m}^{\text {out }}\right\rangle=-a(\langle\hat{k}, \hat{l}\rangle=$ $\langle\hat{k} \hat{l}\rangle-\langle\hat{k}\rangle\langle\hat{l}\rangle)$ and $b=2\left[\left(\Delta \hat{X}_{c}^{\text {out }}\right)^{2}-\left(\Delta \hat{X}_{m}^{\text {out }}\right)^{2}\right]$. For the case of a symmetric distribution in both quadratures, $\Delta \hat{X}_{m}^{\text {out }}=\Delta \hat{P}_{m}^{\text {out }}, \Delta \hat{P}_{c}^{\text {out }}=\Delta \hat{X}_{c}^{\text {out }}$, one obtains $g_{x}=-g_{p}$. We assume the initial state of the light field to be the vacuum state, $\Delta \hat{X}_{c}^{i n}=\Delta \hat{P}_{c}^{i n}=1 / 2$, and that of the mirror to be a thermal state with mean excitation number $n_{b, 0}$, so that $\Delta \hat{X}_{m}^{i n}=\Delta \hat{P}_{m}^{i n}=\sqrt{n_{b, 0} / 2+1 / 4}$.

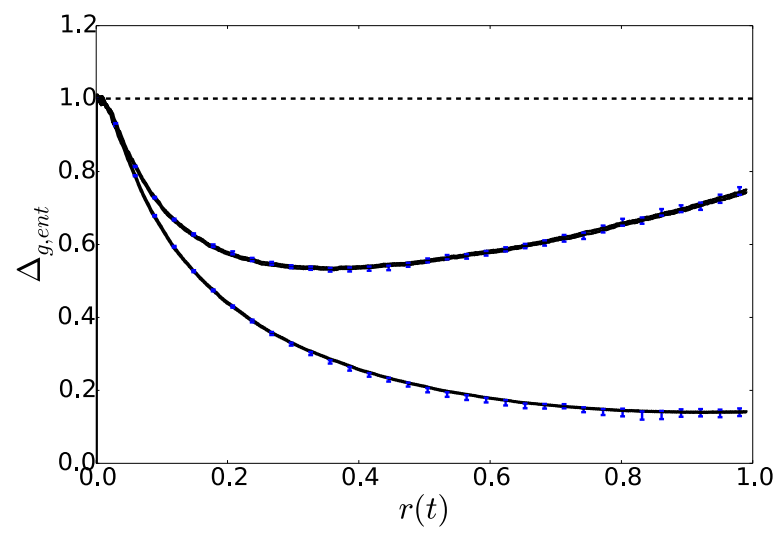

Figure 2. The entanglement signature $\Delta_{g, e n t}$ is plotted versus $r(t)$, for an initial mechanical occupation number $n_{b, 0}=0.7$ and two values of heat bath occupation $\bar{n}_{b, t h}=$ 0.7 (lower), 112(upper) for the oscillator. The cavity bath is assumed a vacuum, i.e. $n_{a, 0}=\bar{n}_{a, t h}=0$. Here the laser driving uses a square pulse shape $(\varepsilon(t)=1 / \sqrt{\tau})$. Solid lines represent the truncated Wigner results with 80000 trajectories. Error bars represent sampling errors using the same trajectory numbers but with the exact positive P-representation method.

The resulting predictions for entanglement detected by the asymmetric witnesses (8) are plotted in Fig. 2.

Using these entanglement signatures, we have simulated the robust asymmetric EPR paradox recently presented in Ref. [38, but for the full standard optomechanical model, without any assumptions. A graph of the predicted the quantum entanglement with low and high temperature is given in Fig. 2. These calculations completely simulate recent experiments on Si optomechanical crystal structures [2. They also demonstrate excellent agreement between the exact positive P-representation and the approximate Wigner method for these parameter values.

The physical interpretation of these results is that for any given initial mechanical oscillator occupation number $n_{b, 0}$, we can always obtain entanglement for $r=$ $\int_{0}^{t} G\left(t^{\prime}\right) d t^{\prime}>0$, provided one uses the asymmetric criteria (8) and selects an optimal choice of gain factor $g(9)$. This means we can detect entanglement in the presence of thermal mechanical decoherence, without the need to use laser cooling to reduce the value of $n_{b, 0}$. However, the minimum value $r_{0}$ required for entanglement detection depends on the accuracy achieved in selecting the gain factors $g$.

Figure 2 indicates entanglement at a temperature $T_{\text {bath }}=20 K\left(\bar{n}_{b, t h}=112\right)$, provided the oscillator is pre-cooled to $T_{0}=200 \mathrm{mK}\left(n_{b, 0} \sim 0.7\right)$. This is sensitive to the occupation number $\bar{n}_{b, t h}$ of the mechanical heat bath, but is more robust to thermal effects than using the symmetric criterion in Ref. 19.

EPR correlations and one-way steering. To signify the EPR steering paradox, it is not sufficient to simply demonstrate entanglement. Here, we present realistic 


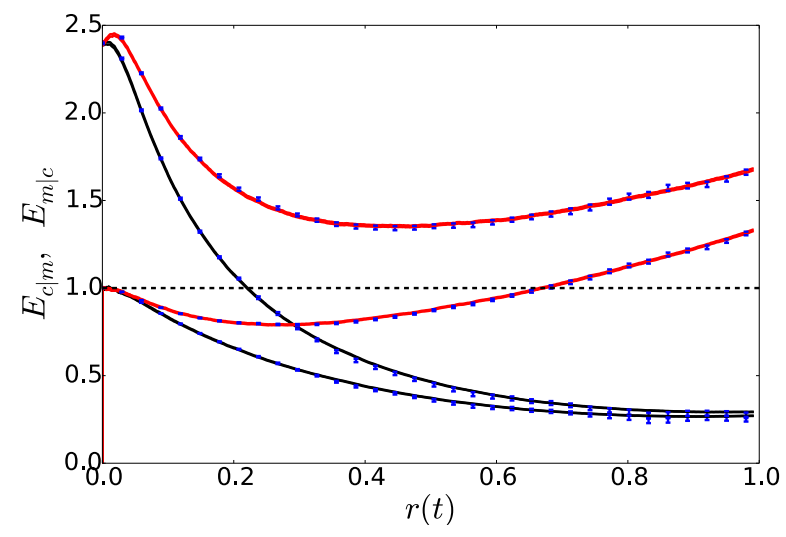

Figure 3. (Color online) Quantum EPR-steering versus squeezing parameter $r$, for the same conditions used in Fig. 2 . The two black curves are for $T_{b a t h}=200 \mathrm{mK}\left(\bar{n}_{b, t h}=0.7\right)$ and the two solid curves are for a higher temperature heat bath with $T_{\text {bath }}=20 K\left(n_{b, t h}=112\right)$. For lower bath temperatures, with a squeezing parameter $r \leq r_{0}, E_{c \mid m}<1$ (lower black) means that only the mirror can steer the optical system, while for $r>r_{0}, E_{m \mid c}<1$ (upper black curve) means that both the mirror and the pulse can steer the other remote subsystem. For a higher temperature heat bath, only one-way steering is possible. The presence of the thermal mechanical excitation makes the steering of the oscillator by the pulse, a more difficult challenge experimentally.

predictions for the steering, optimized for $g$, using phasespace quantum simulations. A simple way to determine if an EPR steering paradox is realized is by testing whether a Heisenberg uncertainty relation is "violated" by inferring uncertainties under the assumptions of local realism (LR), so that 10, 44]

$$
\begin{aligned}
E_{m \mid c} & =4 \Delta_{\text {inf }} \hat{X}_{m}^{\text {out }} \Delta_{\text {inf }} \hat{P}_{m}^{\text {out }} \\
& =4 \Delta\left(\hat{X}_{m}^{\text {out }}+g_{x} \hat{X}_{c}^{\text {out }}\right) \Delta\left(\hat{P}_{m}^{\text {out }}+g_{P} \hat{P}_{c}^{\text {out }}\right)<1
\end{aligned}
$$

Here $\Delta_{\text {inf }} \hat{X}_{m}^{\text {out }}, \Delta_{\text {inf }} \hat{P}_{m}^{\text {out }}$ are the inferred uncertainties, and the optimal scale factors are given as $g_{x}=$ $-\left(\left\langle\hat{X}_{m}^{\text {out }}, \hat{X}_{c}^{\text {out }}\right\rangle+\left\langle\hat{X}_{c}^{\text {out }}, \hat{X}_{m}^{\text {out }}\right\rangle\right) / 2\left(\Delta \hat{X}_{c}^{\text {out }}\right)^{2}$ and $g_{p}=$ $-\left(\left\langle\hat{P}_{m}^{\text {out }}, \hat{P}_{c}^{\text {out }}\right\rangle+\left\langle\hat{P}_{c}^{\text {out }}, \hat{P}_{m}^{\text {out }}\right\rangle\right) / 2\left(\Delta \hat{P}_{c}^{\text {out }}\right)^{2}$. We note that this does not violate the standard Heisenberg relation, which does not use inferred values. For a paradox achieved by condition $(10)$, we can also conclude that pulse $c$ can "steer" the mechanical oscillator $m$ [17, 18].

A thermal barrier exists for this paradox. Figure 3 shows that the mechanical oscillator is steerable by the optical pulse when $r>r_{0}$, where a minimum strength $r_{0}$ of the squeezing parameter required, for a given $n_{b, 0}$. A thermal barrier existing means that a threshold level of pulse-oscillator interaction is required for a given thermal occupation $n_{b, 0}$ of the oscillator.

An EPR paradox can be shown the other way, by the criterion

$$
\begin{aligned}
E_{c \mid m} & =4 \Delta_{\text {inf }} \hat{X}_{c}^{\text {out }} \Delta_{\text {inf }} \hat{P}_{c}^{\text {out }} \\
& =4 \Delta\left(\hat{X}_{c}^{\text {out }}+g_{x} \hat{X}_{m}^{\text {out }}\right) \Delta\left(\hat{P}_{c}^{\text {out }}+g_{p} \hat{P}_{m}^{\text {out }}\right)<1 .
\end{aligned}
$$

Figure 3 shown that this is possible for any value of initial thermal noise $n_{b, 0}$, and for any squeezing parameter $r>0$. This means that there is no equivalent thermal barrier for the optical pulse "steered" by the measurements made on the mechanical oscillator. This effect is possible because the pulse is not thermally excited. We also find that $E_{c \mid m}$ is less sensitive to mechanical decoherence. The reason for this is that we can select optimal gain values $g$ to reduce the effect of the initial thermal noises $n_{b, 0}$ and the heat bath $n_{b, t h}$.

This simulation of the full quantum dynamics not only validates previous analytic results but includes an analysis of the full standard optomechanical Hamiltonian, rather than an idealized parametric model. For a squeezing parameter $r \leq r_{0}$, the only EPR paradox possible is that which verifies the steering of the optical system by the measurements on the mechanical one, i.e. "oneway steering" [38, 45, 47] is predicted. For $r>r_{0}$, "twoway steering" becomes possible, meaning that both mirror and pulse can steer the other subsystem. Clearly, the asymmetry of the steering is due to the asymmetry of the thermal effects on the two systems. The result suggests a fundamental physical principle: changes to a massive system as a result of an action of measurement at a distant site will be inhibited by thermal noise.

\section{Summary}

Optomechanics presents a challenge for exact quantum simulations. It combines a range of occupation numbers and time-scales with non-equilibrium and nonlinear open system quantum dynamics. These results demonstrate that the exact positive-P representation approach is able to give a first principles simulation of the standard model [41]. For the current parameters simulated here, the truncated Wigner approach is also reliable, although this method needs to be verified by more precise methods like the positive- $\mathrm{P}$ approach at large couplings.

We find that probabilistic phase-space simulations of optomechanics are practical and can be carried out with negligible sampling error. An important consequence is that previously used approximations appear unnecessary. For example, our simulations employed square pulses where the adiabatic approximation fails completely - yet there is still very strong entanglement and EPR steering in the simulations. Unlike number-state based approaches, our method can be readily scaled up to study multipartite systems with many oscillator modes [37.

Acknowledgments. - We acknowledge support from the Australian Research Council via Discovery and DECRA grants. 
[1] A. D. O'Connell et al., Nature 464, 697 (2010).

[2] J. Chan et al., Nature 478, 89 (2011).

[3] S. Groblacher et al., Nature Physics 5, 485-488 (2009).

[4] J. D. Teufel et al., Nature 475, 359 (2011); 471, 204 (2011).

[5] A. Safavi-Naeini et al, Phys. Rev. Lett. 108, 033602 (2012).

[6] N. Brahms et al., Phys. Rev. Lett. 108, 133601 (2012).

[7] T. J. Kippenberg and K. J. Vahala, Science 321, 1172 (2008).

[8] M. Aspelmeyer et al., J. Opt. Soc. Am. B 27, A189 (2010).

[9] A. Einstein, B. Podolsky, and N. Rosen, Phys. Rev. 47, 777 (1935).

[10] M. D. Reid, Phys. Rev. A 40, 913 (1989).

[11] E. Schrödinger, Naturwissenschaften 23, 844 (1935).

[12] V. Giovannetti, S. Mancini and P. Tombesi, Europhys. Lett. 54, 559 (2001).

[13] S. Mancini, V. Giovannetti, D. Vitali and P. Tombesi, Phys. Rev. Lett. 88, 120401 (2002).

[14] W. Marshall, R. Simon, R. Penrose and D. Bouwmeester, Phys. Rev. Lett., 91, 130401 (2003).

[15] Max Born, Born-Einstein Letters, 1916-1955: Friendship, Politics and Physics in Uncertain Times (Palgrave MacMillan, 2005).

[16] E. Schroedinger, Proc. Cambridge Philos. Soc. 31, 555 (1935); Proc. Cambridge Philos. Soc. 32, 446 (1936).

[17] H. M. Wiseman, S. J. Jones, and A. C. Doherty, Phys. Rev. Lett. 98, 140402 (2007); S. J. Jones, H. M. Wiseman and A. C. Doherty, Phys. Rev. A 76, 052116 (2007).

[18] E. G. Cavalcanti et al., Phys. Rev. A 80, 032112 (2009).

[19] S. G. Hofer et al., Phys. Rev. A 84, 052327 (2011).

[20] P. D. Drummond and C. W. Gardiner, J. Phys. A: Math. Gen. 13, 2353 (1980). We note that growth of sampling errors in time limit this approach in undamped cases.

[21] M. R. Vanner et al., Proc. Nat. Ac. Sc. 108, 16182 (2011).

[22] I. Pikovski et al., Nat. Phys. 8, 393 (2012).

[23] L. M. Duan, G. Giedke, J. I. Cirac, and P. Zoller, Phys. Rev. Lett. 84, 2722 (2000).

[24] E. P. Wigner, Phys. Rev. 40749 (1932); J.E. Moyal, Proc. Camb.e Phil. Soc. 45, 99 (1949); R. Graham, in Springer Tracts in Modern Physics: Quantum Statistics in Optics and Solid-State Physics, edited by G. Hohler,
(Springer, New York, 1973).

[25] M. J. Steel et al., Phys. Rev. A 58, 4824 (1998).

[26] A. Sinatra, C. Lobo, and Y. Castin, J. Phys. B 35, 3599 (2002).

[27] P. D. Drummond and A. D. Hardman, Europhys. Lett. 21, 279 (1993).

[28] S. Chaturvedi, K. Dechoum, and P. D. Drummond, Phys. Rev. A 65, 033805 (2002).

[29] K. Dechoum et al., Phy. Rev. A 70, 053807 (2004).

[30] M.D. Reid, P.D. Drummond, Phys. Rev. A 40, 4493 (1989), P.D. Drummond, M.D. Reid, Phys. Rev. A 41, 3930 (1990).

[31] Max Ludwig, Björn Kubala and Florian Marquardt, New Journal of Physics 10095013 (2008); Max Ludwig, Amir H. Safavi-Naeini, Oskar Painter, and Florian Marquardt, Phys. Rev. Lett. 109, 063601 (2012).

[32] Andreas Kronwald, Max Ludwig, and Florian Marquardt, Phys. Rev. A 87, 013847 (2013);

[33] Liu Qiu, Lin Gan, Wei Ding, and Zhi-Yuan Li, J. Opt. Soc. Am. B 30, 1683 (2013).

[34] S. J. Carter, M. D. Reid, R. M. Shelby and P. D. Drummond, Phys. Rev. Letters 58, 1841 (1987).

[35] Seth Lloyd, Science 273, 1073 (1996).

[36] Bogdan Opanchuk, Laura Rosales-Zárate, Margaret D. Reid, Peter D. Drummond, arXiv:1301.6305

[37] Jasper Chan, Amir H. Safavi-Naeini, Jeff T. Hill, Sean Meenehan, and Oskar Painter, Applied Phys. Letts. 101, 081115 (2012).

[38] Q. Y. He and M. D. Reid, Phys. Rev. A 88, 052121 (2013).

[39] V. B. Braginsky and A. Manukin, Sov. Phys. JETP 25, 653 (1967).

[40] A. Dorsel et al., Phys. Rev. Lett. 51, 1550 (1983).

[41] A. F. Pace, M. J. Collett, D. F. Walls, Phys. Rev. A 47, 3173 (1993). This widely used model incorporates the Markovian and small-displacement approximations, which are typically very well satisfied.

[42] B. Yurke, Phys. Rev. A 32, 300 (1985).

[43] C. W. Gardiner and M. J. Collett, Phys. Rev. A 31, 3761 (1985); M. J. Collett and D. F. Walls, ibid. 32, 2887 (1985).

[44] M. D. Reid et al., Rev. Mod. Phys. 81, 1727 (2009).

[45] V. Händchen et al., Nature Photonics 6, 596 (2012).

[46] S. Midgley, A. J. Ferris and M. K. Olsen, Phys. Rev. A 81, 022101 (2010).

[47] K. Wagner et al., arXiv:1203.1980 [quant-ph]. 